\title{
Nontoxic membrane translocation peptide from protamine, low molecular weight protamine (LMWP), for enhanced intracellular protein delivery: in vitro and in vivo study
}

\author{
Yoon Jeong Park, * Li-Chien Chang, ${ }^{\dagger}$ Jun Feng Liang, ${ }^{\star}$ Cheol Moon, ${ }^{\S}$ Chong-Pyoung Chung, ${ }^{\natural}$ \\ and Victor C. Yang ${ }^{\S}$ \\ *Craniomaxillofacial Reconstructive Science Major and "Department of Periodontology, School \\ of Dentistry and Intellectual Biointerface Engineering Center, Seoul National University, Seoul, \\ South Korea; ${ }^{\dagger}$ School of Pharmacy, National Defense Medical Center, Taipei, Taiwan; \\ Department of Chemistry and Chemical Biology, Stevens Institute of Technology, Hoboken, \\ New Jersey, USA; and ${ }^{\S}$ College of Pharmacy, University of Michigan, Ann Arbor, Michigan, \\ USA
}

Corresponding author: Yoon Jeong Park, School of Dentistry and Intellectual Biointerface Engineering Center, Seoul National University, 28-2 Yongon-Dong, Chongno-Ku, Seoul 110749, South Korea. E-mail: parkyj@snu.ac.kr

\section{ABSTRACT}

Naturally derived, nontoxic peptides from protamine by the authors, termed low molecular weight protamines (LMWPs), possess high arginine content and carry significant sequence similarity to that of TAT, by far the most potent protein transduction domain peptide. Therefore, it was hypothesized that these LMWPs would also inherit the similar translocation activity across the cell membrane, which enables any impermeable species to be transduced into the cells. LMWPs were prepared by enzymatic digestion of protamine, examined their capability of transducing an impermeable protein toxin into the tumor cells by chemical conjugation, and determined cytotoxicity of transduced protein toxin (e.g., gelonin) against cancer cell lines and a tumor-bearing mouse. In vitro results showed that LMWPs could indeed translocate themselves into several mammalian cell lines as efficiently as TAT, thereby transducing impermeable gelonin into the cells by chemical conjugation. In vivo studies further confirmed that LMWP could carry an impermeable gelonin across the tumor mass and subsequently inhibit the tumor growth. In conclusion, the presence of equivalent cell translocation potency, absence of toxicity of peptide itself, and the suitability for low-cost production by simple enzymatic digestion could expand the range of clinical applications of LMWPs, including medical imaging and gene/protein therapies.

Key words: LMWP • PTD • TAT $・$ gene therapy $\bullet$ medical imaging

The potential for intracellular molecular imaging and therapeutic use of proteins, peptides, and oligonucleotides has been far limited by poor bioavailability as a result of the cellimpermeable nature of these compounds. Recently, several small regions of proteins termed protein transduction domains (PTDs), including peptides from human immunodeficiency 
virus TAT protein (1-3), Antennapedia homeodomain transcription factor (4), and Herpes simplex virus type 1 VP22 (5), have received significant and widespread attention within the pharmaceutical and medical societies, as a result of their unprecedented ability to deliver macromolecules into living cells. By covalently linking these PTDs to a variety of species including hydrophilic fluorescent probes $(6)$, macromolecular proteins $(1-3,7)$, nano-carriers of $\sim 45 \mathrm{~nm}$ in size $(8,9)$, and liposomes $(10)$, these peptides were shown to be capable of translocating such attached species into all cell types, in vitro and in vivo. The efficiency of PTD-mediated cell-translocation could not be matched by any of the existing transcellular pathways including the often-used, receptor-mediated endocytosis (11). Added to this attractive feature in potential biomedical applications is that cell internalization mediated by PTDs did not cause membrane perturbation or damage $(7,12)$. As it has been known to be a receptorindependent process $(1-3,7,12)$, in principle and practice, all cell types would be susceptible to PTD-mediated cell transduction.

We recently prepared several nontoxic, arginine-rich peptides termed low molecular weight protamine (LMWP) by enzymatic digestion from natural protamine (13-16). These LMWPs were derived directly from native protamine by enzymatic digestion with thermolysin. It is most important that unlike other cationic proteins/peptides, these LMWPs elicited only minimal complement activation and no detectable hypotensive or toxic responses in dogs (16). Furthermore, in vitro and animal studies also demonstrated that LMWPs were neither antigenic (17) nor mutagenic (18). As these LMWP peptides possess a high arginine content and carry a significant sequence similarity $(14,19)$ to that of $\operatorname{TAT}_{47-57}(7)$, the most extensively studied PTD to date, it is hypothesized that they would possess a similar cell-translocating, potency-like TAT. In addition, the toxicity profiles of LMWP have already been thoroughly established (16-18). Also, unlike the other PTDs, LMWP could be produced from native protamine in mass quantities with relative ease and low costs.

In this paper, we demonstrated that LMWP is able to confer protein transduction of a wide variety of cell types with an efficiency that is at least equivalent to the TAT PTD for molecular imaging and therapeutic purpose. We also presented preliminary in vivo findings of the feasibility that LMWP could be used as a transmembrane carrier to deliver gelonin, a cellimpermeable protein toxin, into tumors for cancer treatment.

\section{MATERIALS AND METHODS}

\section{Materials}

Salmine protamine, thermolysin, gelonin, fluorescein isothiocyanate (FITC), rhodamine, and 3(4,5-dimethylthiazol-2-yl)-2,5-diphenyltetrazolium bromide (MTT) were purchased from Sigma Chemical Co. (St. Louis, MO, USA). Alexa Fluoro488-phalloidin and rhodamine-phalloidin were from Molecular Probes (Eugene, OR, USA). Fetal bovine serum (FBS), phosphate-buffered saline (PBS), 0.25\% (w/v) trypsin-EDTA, RPMI-1640 medium, Dulbecco's modified essential medium (DMEM), $\alpha$-minimum essential medium $(\alpha-\mathrm{MEM})$, and penicillin/streptomycin antibiotics were from Gibco-BRL (Gaithersburg, MD, USA). N-succinimidyl-S-acetylthioacetate (SATA) and bifunctional cross-linkers, 1-ethyl-3-(3-dimethylaminopropyl) carbodiimide hydrochloride (EDC) and N-succinimidyl-3-(2-pyridyldithio) proprionate (SPDP), were obtained from Pierce Inc. (Rockford, IL, USA). The peptide containing the $\mathrm{TAT}_{47-57}$ (CYGGYGRKKRRQRRRR) sequence was synthesized at the Protein Core Facility, University 
of Michigan (Ann Arbor, MI, USA). All of the solvents used were of analytical grade, and water was distilled and deionized.

\section{Preparation of LMWP peptides}

The enzymatic method used for the preparation of LMWP was described in previous publications (13-19). In brief, thermolysin and protamine were mixed and incubated for $30 \mathrm{~min}$ at room temperature, followed by quenching of thermolysin activity with $50 \mathrm{mM}$ EDTA. Thermolysin was then removed by ultrafiltration using a YM3 membrane (MWCO 3000), and the filtrate was subject to lyophilization. As the lyophilized LMWP preparation contained a mixture of small peptide impurities, it was further purified with a heparin affinity chromatography. A total of five peptides, denoted thermolysin-digested segment of protamine (TDSP)1-5, according to the order of elution from the heparin column, was obtained. The molecular weight and amino acid sequence of each isolated LMWP peptide were determined by matrix-assisted laser desorption/ionization (MALDI) time-of-flight mass spectrometry (MS) analysis, which was performed by the Protein and Carbohydrate Research Center at the University of Michigan. Among the five fractions, TDSP5 (VSRRRRRRGGRRRR) was used in all subsequent studies as a result of its similarity in structure to $\mathrm{TAT}_{47-57}$ peptides.

\section{Preparation of LMWP-protein/peptide conjugates}

To conjugate LMWP to phalloidin, rhodamine-phalloidin ( $2 \mathrm{mg} / \mathrm{ml}$ in ethanol) was mixed with EDC [100 $\mu 10.2 \mathrm{M}$ EDC in dimethylformamide (DMF) to $1 \mathrm{ml}$ phalloidin solution] for $5 \mathrm{~h}$. Hydrolytic product of unreacted EDC was precipitated by excess of ethanol/methylene chloride and removed. The rhodamine-phalloidin-EDC conjugate was then reacted with LMWP (tenfold molar excess of LMWP:phalloidin). The solution was subjected to incubation stirring overnight at $4{ }^{\circ} \mathrm{C}$. To conjugate gelonin to LMWP, LMWP needed to be modified to bring reactive sulfhydryl moiety.

The LMWP (TDSP5, VSRRRRRRGGRRRR) was modified at its N-terminus by SATA and hydroxylamine to create a free sulfhydryl group. In brief, LMWP $(5 \mathrm{mg} / \mathrm{ml}$ in phosphate buffer, $\mathrm{pH}$ 7.4) was mixed with a tenfold molar excess of SATA, dissolved in dimethyl sulfoxide (DMSO), and reacted for $2 \mathrm{~h}$ at room temperature. SATA-modified LMWP was then treated with hydroxylamine to cleave the protected acetyl group of SATA to bear the free sulfhydryl group at the N-terminus of LMWP. The content of sulfhydryl in the LMWP was measured using Ellman's reagent, as described elsewhere $(20,21)$. Gelonin $(1 \mathrm{mg} / \mathrm{ml}$ in water) was mixed with SPDP (40 $\mu 10.1 \mathrm{M}$ SPDP in ethanol to $1 \mathrm{ml}$ protein solution) and stirred while incubated at room temperature for $5 \mathrm{~h}$. The hydrolytic product of unreacted SPDP was removed from the solution by size exclusion chromatography (Hi-Trap desalting column), and fractions containing gelonin were pooled. The gelonin solution was then mixed, under reducing conditions, with sulfhydryl (SH)-LMWP (tenfold molar excess of SH-LMWP:protein) and HEPES buffer ( $\mathrm{pH} 8.3$ ) to give an overall HEPES molarity of $50 \mathrm{mM}$. The solution was subjected to incubation stirring overnight at $4^{\circ} \mathrm{C}$. Conjugated LMWP-protein was separated from unconjugated-free protein and free LMWP by heparin-affinity chromatography (Hi-Trap heparin-affinity column) and pooled. Pooled LMWP-protein conjugate was concentrated, and its concentration was determined. The conjugated LMWP-protein was examined by sodium dodecyl sulfate-polyacrylamide gel electrophoresis (SDS-PAGE) and MALDI-MS to determine the number of LMWP moieties per protein. The molar incorporation of LMWP:protein was calculated to be $\sim 1: 1$. 


\section{Fluorescence labeling}

LMWP and $\mathrm{TAT}_{47-57}$ peptides were labeled with FITC at their N-terminals. In brief, the peptide solution ( $\mathrm{pH}$ 9.3, carbonate buffer) was reacted in a 1:2 molar ratio with a FITC solution (in DMF) overnight in the dark at room temperature. Reaction was monitored by high-pressure liquid chromatography (HPLC) of the absorbance change at $215 \mathrm{~nm}$ of the peptide peak. The labeled peptides were purified by HPLC (purity $>95 \%$ ), lyophilized, and then stored at $-20^{\circ} \mathrm{C}$ in the dark until further use. As LMWP-phalloidin was already labeled with rhodamine, no further labeling procedure was conducted. However, LMWP-gelonin conjugate was labeled with rhodamine isothiocyanate and purified by a similar method described above, lyophilized, and stored at $-20^{\circ} \mathrm{C}$ in the dark until further use.

\section{Inhibition of protein synthesis by LMWP-gelonin conjugates in a cell-free translational assay}

The effect of conjugation chemistry on the ribosomal-inactivating properties of LMWP gelonin or TAT gelonin was examined using a rabbit reticulocyte lysate assay kit from Amersham Pharmacia (Little Chalfont, UK), followed by the manufacturer's protocol.

\section{Cell lines and culture}

The 293T, HeLa human cervical carcinoma, CT-26 colon adenocarcinoma cell line, human MCF-7 breast carcinoma cell lines, MG 63 osteoblast cell line, and NIH3T3 fibroblast cell lines were obtained from American Type Culture Collection (Manassas, VA, USA). They were cultured in $\alpha$-MEM medium (293T, HeLa, MG63, NIH3T3) or RPMI 1640 (for CT-26, MCF-7), supplemented with $10 \% \mathrm{FBS}, 100$ units/ml penicillin-streptomycin mixture, and $2.2 \mathrm{mg} / \mathrm{ml}$ sodium bicarbonate at $37^{\circ} \mathrm{C}$ in humidified $5 \% \mathrm{CO}_{2}$.

\section{Flow cytometric analyses}

The cells were seeded at a density of $1 \times 10^{6}$ cells per well in six-well plates in $1.5 \mathrm{ml}$ culture medium. One day later, the cells were washed and incubated with FITC-labeled peptides $(0.2$ $\mu \mathrm{M}$ ) for $30 \mathrm{~min}$ at $37^{\circ} \mathrm{C}$ in humidified $5 \% \mathrm{CO}_{2}$. To study cell internalization in the presence of serum, the peptides were dissolved in DMEM in the absence of serum, followed by the addition of $10 \%$ FBS. After incubation, cells were washed with PBS, treated extensively with trypsinEDTA to remove a surface-bound, FITC-labeled peptide, and washed again. The cells were then fixed with $1 \%$ paraformaldehyde and were washed with PBS. Analysis was conducted on a FACSCaliber flow cytometer (Becton Dickinson, San Jose, CA, USA), equipped with a 488-nm air-cooled argon laser. The filter settings for emission were 530/30 nm bandpass (FL1) for FITC. The fluorescence of 5000 vital cells was acquired, and data were visualized in a logarithmic mode.

\section{Confocal microscopic observation of peptide and conjugate internalization}

Cells were plated on Lab-Tek (four-well) chambered coverglasses at a density of $1 \times 10^{4}$ cells/coverglass and incubated at $37^{\circ} \mathrm{C}$ in humidified $5 \% \mathrm{CO}_{2}$. After complete adhesion, the culture medium was removed. The FITC-labeled LMWP, TAT, or LMWP-rhodamine-labeled phalloidin conjugate was then added to the cells at a final peptide concentration of $0.2 \mu \mathrm{M}$. Following $30 \mathrm{~min}$ incubation at $37^{\circ} \mathrm{C}$ in humidified $5 \% \mathrm{CO}_{2}$, cells were washed extensively at 
the cell surface as described elsewhere and fixed in 1\% (w/v) paraformaldehyde in PBS for 20 min at room temperature. As well as the labeled peptides, LMWP-gelonin, TAT-gelonin, or gelonin was applied to the cells, incubated for $30 \mathrm{~min}$, and fixed with $1 \%$ paraformaldehyde. The fixed cells were washed again with PBS and kept at $4^{\circ} \mathrm{C}$ for at least $1 \mathrm{~h}$ before being examined by confocal microscopy. The cells treated with rhodamine-labeled phalloidin-LMWP conjugates were observed by confocal microscopy without a fixation step. Confocal laser-scanning microscopy was carried out using an inverted LSM 510 laser-scanning microscope (Carl Zeiss, Gottingen, Germany) equipped with a Plan-Apochromat $63 \times 1.4$ N.A. or $40 \times 1.4$ N.A. lens. The laser was set at 488 (blue) and 543 (yellow) to produce the excitation wavelength for fluorescein and rhodamine, respectively. Z-series were taken of a 1- to 2 - $\mu \mathrm{m}$ optical section at 2 $\mu \mathrm{m}$ intervals.

\section{Cytotoxicity assay}

Evaluation of the cytotoxicity of a series of LMWP peptides was conducted using the MTT assay. In general, the $293 \mathrm{~T}$ cells were seeded at a density of $1.0 \times 10^{4}$ cells/well in the 96-well, flatbottomed microassay plate (Falcon Co., Becton Dickinson, Franklin Lakes, NJ, USA) and incubated for $24 \mathrm{~h}$. The LMWP sequences (TDSP2, TDSP3, TDSP4, TDSP5) or polyethylenimine (PEI), as a control, were then added in varying concentrations to the culture media, and the mixture was incubated for another $72 \mathrm{~h}$ at $37^{\circ} \mathrm{C}$. At the end of the experiment, the medium was replaced with $200 \mu \mathrm{l}$ fresh DMEM without serum, and $120 \mu \mathrm{l} 2 \mathrm{mg} / \mathrm{ml}$ MTT solution in PBS was then added. After incubation for an additional $4 \mathrm{~h}$ at $37^{\circ} \mathrm{C}$, the MTTcontaining medium was removed, and $200 \mu \mathrm{DMSO}$ was added to dissolve the formazan crystal formed by live cells. Absorbance was measured at $570 \mathrm{~nm}$. The cell viability (\%) was calculated according to the following equation:

$$
\text { Cell viability }(\%)=\left[\mathrm{OD}_{570(\text { sample })}\right] /\left[\mathrm{OD}_{570(\text { control })}\right] \times 100,
$$

where the optical density $(\mathrm{OD})_{570 \text { (sample) }}$ and $\mathrm{OD}_{570 \text { (control) }}$ represented measurements from wells treated with each peptide sample and that without peptide treatment, respectively.

\section{Bioassay of LMWP-gelonin conjugates}

The bioassay of conjugated gelonin with LMWP or TAT in vitro for anticancer effect against the CT-26 colon adenocarcinoma cell line was conducted by the same method of MTT assay described above. In brief, cells grown with $75 \%$ confluency of $1.0 \times 10^{4}$ cells/well were incubated with various concentrations of LMWP-gelonin or TAT-gelonin conjugates. As a control, native gelonin was served. Cell proliferation was measured over $48 \mathrm{~h}$, on which cell culture medium was removed and replaced with fresh PBS containing $2 \mathrm{mg} / \mathrm{ml}$ MTT. The absorbance of the MTT-treated cell culture medium was measured at $570 \mathrm{~nm}$ after addition of DMSO, and the survival ratio was determined by the ratio of the absorbance of sample-treated cells to that of cells with no treatment, as described in the previous section.

\section{In vivo antitumor effect of LMWP-gelonin conjugates}

Five-week-old female BALB/c mice were purchased and housed in the Animal Care Facilities. Mice were maintained on ad libitum rodent feed and water at room temperature, $40 \%$ humidity. All mice were acclimated for at least 1 week before tumor implantation. All studies were 
performed in accordance with an animal protocol approved by the University of Michigan Institutional Animal Care. Subcutaneous (s.c.) tumor-bearing BALB/c mice were used as an animal model for evaluation of LMWP gelonin for its anticancer activity. To generate tumors, 5week-old $\mathrm{BALB} / \mathrm{c}$ mice were s.c.-injected in the right-hind flank with $100 \mu \mathrm{l}$ single-cell suspension containing $4 \times 10^{6} \mathrm{CT}-26$ cells. Tumor was measured with vernier calipers, and volumes were calculated according to the following formula: Volume $=0.52 \times \mathrm{W}^{2} \times \mathrm{L}$, where $\mathrm{W}$ and $\mathrm{L}$ represent the width and length, respectively. Treatment of the tumors started 2 weeks after tumor implantation when their size reached $100-150 \mathrm{~mm}^{3}$. Test compounds included PBS solution (control); gelonin (100 $\mu \mathrm{g})$; LMWP-gelonin conjugate (100 $\mu \mathrm{g}$ gelonin equivalence); mixture of free LMWP and gelonin $(100 \mu \mathrm{g})$; and TAT-gelonin conjugates $(100 \mu \mathrm{g}$ gelonin equivalence). Each experimental group contained eight mice. The mice were treated with those samples $(50 \mu \mathrm{lvol})$ for nine times over 15 days by peritumoral injection. The tumor volume was measured at 3- to 4-day intervals. At the end of the observation, mice were killed and autopsied; tumors were removed, weighed, and fixed with formalin.

\section{RESULTS}

\section{Cell translocation functions of LMWPs}

LMWP fragments were derived from native protamine by thermolysine digestion according to a previously published procedure (13-19). Five different fractions, denoted as TDSP1-5, depending on their elution order from a heparin-affinity column, were isolated and purified (Fig. $\underline{1 A}$ ). Except for TDSP1, which possessed less than six arginine residues required for cell transduction (19), the cell internalization activity of TDSP2, $-3,-4$, and -5 was examined. All of the studied LMWP peptides displayed sufficient cellular uptake at 30 min incubation with the increase of the arginine content in these peptides (Fig. 1B). The TDSP5 peptide further demonstrated the enhanced cellular uptake, as cells treated with TDSP5 exhibited the highest fluorescence intensity, and was largely localized in the cytoplasm of HeLa cells (Fig. 1C). Similar results were also obtained by using other cell line types including 293T, CT-26, human MCF-7 cells, fibroblast, as well as MG 63 osteoblasts. As seen, native protamine showed almost identical translocalization efficiency to that of TDSP5 (Fig. 1B), which was coincident with the previous report, indicating the similar functional capacity of protamine and TDSP5, i.e., heparin neutralization $(13,14)$. To evaluate the effect of serum on the transduction efficiency of LMWP, identical experiments were conducted in cell culture medium containing $10 \%$ FBS (Fig. 2). The efficiency of TDSP5 uptake was not affected by the presence of serum. In addition, these peptides showed no significant cytotoxicity (i.e., $<10 \%$ reduction in cell viability) up to a concentration of $10 \mathrm{mM}$, whereas PEI, a common synthetic-transfecting agent, showed a significant decrease (by $40 \%$ ) of cell viability at or above a concentration of $5.0 \mathrm{mM}$ (Fig. 3).

\section{Transcellular delivery of protein/peptides mediated by LMWP}

To examine if LMWP could be used as a carrier for intracellular delivery of cargo molecules for imaging or therapeutic purpose, phalloidin or gelonin was conjugated to LMWP. Each molecule was conjugated to LMWP by a 1:1 molar ratio (Fig. 4A). MALDI-MS results demonstrated the molecular weight of LMWP-gelonin and gelonin as 31,500 and 29,200, respectively. The difference of the molecular weight between LMWP-gelonin and gelonin was almost identical to the molecular weight of LMWP, one molecule, indicating a 1:1 ratio of the LMWP:gelonin in the conjugate. The heparin column elution profile of the reaction mixture provided three 
representative peaks: unreacted gelonin (first peak), LMWP-gelonin conjugate (second peak), and unconjugated LMWP and the mixture of the conjugate with a higher amount of LMWP attached (third peak). The MALDI-MS showed the first peak as gelonin, the second peak as LMWP-gelonin at a 1:1 molar ratio, and the third peak as the mixture of free LMWP and LMWP-gelonin with higher LMWP attached. We used the sample from the second peak (samples with a 1:1 molar ratio of LMWP and gelonin) for further study. The ability of LMWPgelonin, TAT-gelonin, and gelonin to inhibit translation in a cell-free system was determined by using a rabbit reticulocyte translation assay. The LMWP-gelonin, TAT-gelonin, and gelonin had inhibitory concentration $50 \%\left(\mathrm{IC}_{50}\right)$ values of $\sim 23,15$, and $49 \mathrm{pM}$, respectively, indicating that conjugation of gelonin to LMWP and TAT did not reduce the bioactivity of the toxin component of gelonin.

To compare the translocation activity of LMWP with other known PTDs, TAT was selected as a control and conjugated to gelonin. As shown by the FACS results (Fig. 4B $)$, the ability to translocate gelonin by LMWP and TAT was closely comparable. In both cases, rhodamine labels were clearly detected after 30 min of incubation of these peptides with CT-26 cells. Data from confocal microscopic studies were also in good agreement with the FACS results, displaying a cytoplasmic localization of the TDSP5-gelonin conjugates instead of adsorption to the cell surfaces (Fig. 4C). In addition, the cells treated with LMWP-phalloidin conjugates exhibited quick and obvious cytoskeleton labeling without any permeabilization step of the cell membranes (Fig. 4D, E), and treating with phalloidin could not label cells without permeabilization (data not shown). To further confirm the LMWP-gelonin conjugates actually penetrated into the tumor, rhodamine-labeled gelonin was applied during in vivo studies. The tumor tissues from LMWP-gelonin-treated mice displayed a strong and uniform red staining from rhodamine on the labeled LMWP-gelonin conjugates (Fig. 4F), implicitly confirming the penetration of the LMWP-gelonin conjugate into the tumor tissues. Conversely, mice injected with rhodamine-labeled, free gelonin displayed virtually no or at most, a sporadic, weak staining of rhodamine (Fig. 4G). Therefore, the antitumor effect of LMWP-gelonin was anticipated by the results from the enhanced distribution of the conjugates into the tumor tissues.

\section{In vitro activity of the LMWP-gelonin conjugate in the cells}

Activity of protein conjugate in the cells, cytotoxicity of the gelonin conjugate, for example, was measured using gelonin and LMWP- and TAT-gelonin conjugates against CT-26 colon adenocarcinoma cells in the log-phase culture medium. As expected, gelonin itself did not cause any detectable inhibition of cell growth (Fig. 5), simply as it could not internalize the cells. Coadministration of LMWP or TAT with gelonin also did not yield any cytotoxic effects (data not shown), indicating that without covalent conjugation with LMWP or TAT, gelonin itself was still not able to cross the cell membrane. On the contrary, the TAT- and LMWP-gelonin conjugates were found to be highly cytotoxic, and $\mathrm{IC}_{50}$ values (measured by the MTT assay) were $20-40 \mathrm{pM}$ (Fig. 5). Although TAT-gelonin appeared to yield a slightly lower $\mathrm{IC}_{50}$ value (20 $\mathrm{pM})$ than LMWP-gelonin (39 pM), this difference was relatively insignificant and could attribute to inconsistency in determining the concentrations of the conjugates in the stock solutions. Nevertheless, results in Fig. 5 clearly demonstrated that via a covalent linking, LMWP was able to successfully transduce an otherwise cell-impermeable protein toxin into cancer cells for possible therapeutic purposes. 


\section{In vivo activity of LMWP-gelonin for tumor therapy}

Biological consequences of treating s.c. solid tumors with LMWP-gelonin were assayed after peritumoral injection of test samples (Fig. 6). Pooled data from multiple experiments revealed that the treatment with LMWP-gelonin resulted in a substantial reduction of tumor mass in all mice (Fig. 6A). In contrast, tumors treated with free gelonin were larger than their LMWP gelonin-treated counterparts in all mice. As seen, tumors continued to grow in the control mice injected with PBS solution, and the average mass of excised tumors reached $3.16 \pm 0.65 \mathrm{~g} 4$ weeks after treatment (Fig. $6 B, \underline{C})$. As expected, mice treated with free gelonin did not display regression on tumor growth, as gelonin could not penetrate the tumor. An average tumor weight of $2.63 \pm 0.5 \mathrm{~g}$ was observed 4 weeks after injection of gelonin. This average tumor mass was statistically indistinguishable $(P<0.05)$ from that of the control group, especially considering the inconsistency and inaccuracy involved in counting the volumes of the excised tumors. In a sharp contrast, mice treated with the LMWP-gelonin conjugate displayed a significant regression in tumor growth, as the tumor weight was reduced to an insignificant value of $0.33 \pm 0.12 \mathrm{~g}$. Addition of free LMWP to the gelonin solution did not elicit any effect on tumor regression, as an average tumor mass of $2.74 \pm 0.68 \mathrm{~g}$ was observed 4 weeks after treatment, clearly indicating that only covalent conjugation of LMWP was able to transduce gelonin and present the antitumor effect with the same extent as that by the TAT conjugate $(0.21 \pm 0.04 \mathrm{~g})$.

\section{DISCUSSION}

Drug therapies and medical imaging face the same bottleneck limitation, i.e., difficulty of delivering active agents into cells. Normally, efficient delivery of therapeutic and imaging compounds can be achieved only when such agents are hydrophobic and small, typically in the range of less than 600 daltons $(7,22)$. The most effective means to date to achieve intracellular transfer of biomolecules is by receptor- or transporter-mediated endocytosis. This method, however, suffers from the low-efficiency and incapability to deliver large compounds (e.g., proteins or genes) into living cells. This was the primary reason that interest in macromolecule delivery was heightened dramatically since 1994, when Fawell and co-workers (1) demonstrated that proteins and DNAs chemically linked to the TAT peptide could internalize the cells effectively. In fact, research related to the use of PTDs has currently become one of the hottest pursuits in the medical and pharmaceutical societies, as reflected by the drastic increase in volumes of publications in recent years. As PTD-mediated cell transduction is receptor (transporter)-independent, in principle and practice, all types of compounds (whether they are small, large, hydrophobic, or hydrophilic) are transducible against all cell types (1-12).

Aside from this translocalization capacity, the LMWPs possess two major advantages. First, these LMWP peptides can be derived directly from protamine by using a "separation-free" enzymatic degradation (i.e., via the use of immobilized thermolysin) and a single-step isolation/purification process (i.e., via the use of a heparin-affinity chromatography) and thus, can be produced in mass quantities within a short time duration (e.g., a production rate of 1 $\mathrm{g} /$ week can be achieved based on our own laboratory scale). Second, extensive and conclusive animal studies have been conducted on these LMWP peptides to confirm that they actually possess a significantly reduced antigenicity (17; i.e., the ability of a substance to be recognized by an antibody), mutagenicity (18), and complement-activating activity (16) and other cationic, polymer-associated, hemodynamic/hematologic toxic effects (16) than the parent protamine, a U.S. Food and Drug Administration (Rockville, MD, USA)-approved, widely used clinical drug. 
Furthermore, in a direct comparison of the cytotoxicity between LMWP (i.e., TDSP5) and the currently used transfecting agent, PEI, our results (Fig. 3) showed that PEI induced a far more significant reduction in cell viability than TDSP5, even when it was present in half of the concentration of the latter. Insensitivity of cell uptake by LMWPs to the presence of serum added an attractive feature for their possible in vivo application, as numerous reports had indicated that serum protein would interfere with effective cellular uptake or targeting of a peptide (23).

Results in Fig. 1 showed that the cell-translocation functions of LMWPs were strongly dependent on the arginine content in these peptides. This finding was indeed in good agreement with data reported by other investigators about the synthesized, arginine-rich peptides (12). It was demonstrated that polyarginine peptides possessed a cell translocation activity and internalization pathway similar to those of TAT $(22,24)$. Indeed, it was concluded that the presence of at least six arginine residues was an essential requirement for these peptides to achieve an effective intracellular uptake (22-25). Peptides with an arginine content of eight residues were shown to exhibit the highest cell translocation activity, whereas peptides with over 12 arginines actually displayed a decline of such an activity $(25,26)$. In our results, TDSP5, which contained 10 arginine residues, yielded the highest degree of cellular uptake among all the LMWP peptides, suggesting that there might be a critical and tightly regulated arginine content ( $\sim 10$ residues) to achieve the most efficient translocation across the cell membranes. Results from confocal microscopic studies (Fig. 1B) also confirmed that LMWPs were localized primarily in the cytosol and nucleus, and a small fraction of it was adhered to the cell membranes.

As discussed earlier, the most attractive feature of PTDs is its ability to transduce impermeable species into living cells. This unique, cell-transduction function would render it possible to achieve successful protein and gene delivery and effective in vitro screening of potential therapeutic candidates and elucidate the role of proteins inside the cells by directly targeting specific events throughout the cell cycle. To examine if LMWPs would also possess such important functions, we attempted to deliver a full-length protein, gelonin, or impermeable peptide phalloidin into mammalian cells. Fluorescently tagged phalloidin has been used to label cytoskeleton of cells; however, it by itself, cannot enter the cells, thereby requiring an additional step for membrane permeabilization/fixation to provide an entry channel for phalloidin. When LMWP was conjugated to the phalloidin, in contrast, the conjugates entered into the cytoplasm and labeled the cytoskeleton without any permeabilization/fixation treatment. It should be pointed out that the intracellular target molecules, as well as epitope of target tissues can be labeled and visualized in a real-time manner by introducing the transduction domain peptide such as LMWP, which further will be used in medical molecular imaging. Meanwhile, the primary reason to select gelonin was that gelonin represented the class of hydrophilic, macromolecular drugs whose therapeutic functions were limited by poor cellular uptake. Indeed, despite its potent inhibitory action on protein synthesis, native gelonin was known to yield low cytotoxicity toward tumors as a result of its inability to cross the cell membrane (27-29). This lack of cell-entering ability actually renders gelonin an ideal candidate to test the functions of LMWP via the fluorescence labeling and cytotoxicity-assaying methods. As shown from the FACS and confocal results in Fig. 4, conjugation of a single molecule of LMWP to gelonin was sufficient to translocate the entire conjugate into CT-26 cells. Almost all of the labeled LMWPgelonin conjugates were found inside, specifically in the cytosol of the CT-26 cells (Fig. 4C). In agreement with results reported by other investigators of the TAT-linked protein conjugates (30), further incubation of the LMWP-gelonin conjugate resulted in the localization of the conjugates inside the nucleus (data not shown). This rapid, intracellular gelonin transduction was able to 
evoke significant cytotoxic effects against the CT-26 cells, as a relatively lower $\mathrm{IC}_{50}$ value in the range of 20-40 pM was observed. It is interesting that the $\mathrm{IC}_{50}$ value of the LMWP-gelonin conjugate was nearly identical to that of the TAT-gelonin conjugate but considerably lower than that reported for the antibody-gelonin immunotoxin $(29,31)$. This superior cytotoxicity of LMWP-gelonin was apparently a result of the far more efficient cell-internalization activity of LMWP over the endocytosic activity of an antibody.

To further confirm the cell transduction function of LMWP in vivo, we attempted the delivery of gelonin to BALB/c mice harboring CT-26 solid tumors. As shown from photographs of excised tumors (Fig. 6), 4 weeks after treatment, peritumoral injection of free gelonin failed to elicit any detectable tumor regression (Fig. 6C), as gelonin by itself could not penetrate the tumor. On the contrary, animals receiving administration of the LMWP-gelonin conjugate displayed nearly a complete tumor regression (Fig. 6C), as the recovered tumor mass was almost negligible considering the difficulty and inaccuracy in extracting these tumors. Indeed, tumors in these animals actually collapsed into a scabrous tissue plug, which later dropped off to reveal a scar tissue at the spot of the previous tumor implantation site. Coadministration of free LMWP also failed to trigger any tumor regression (Fig. 6C), as without a covalent linkage, LMWP was not able to transduce gelonin. It should be pointed out that this extraordinary efficiency in tumor regression could be partly a result of the cytosol localization of the LMWP-linked gelonin, which was then able to exert its maximum antitumor functions by binding to the ribosomes in the cytosol.

The unprecedented and unparalleled power of PTDs to transduce macromolecules into cells has drawn speculation of whether this phenomenon is for real. Indeed, several investigators (27-29) have suggested that PTD-mediated cell transduction is actually a technical artifact that occurs during the cell-fixation process. However, this artifact has recently been avoided by extensive washing of the cell surface using enzyme, and the current study followed the same procedure as conducted by other studies. Along with these in vitro transduction results, the in vivo tumor transduction of protein toxins was in good agreement with the transduction by the LMWP; it is more important that no cell fixation process was involved during the animal studies. Several hypotheses have been suggested to account for the mechanisms of PTD-mediated cell transduction including a formation of hydrogen bonds between the guanidine head group in the arginine residues with phospholipids on the cell membrane (32) and interaction of the arginine residues with the extracellular matrix glycosaminoglycans, such as heparan sulfate or chondroitin sulfate (33). Albeit unclear, the LMWP-mediated cell transduction could be well-related to one or all of these mechanisms.

In summary, in vitro and in vivo results have demonstrated that LMWP, especially TDSP5, is as potent a cell-transduction peptide as any existing PTD, including TAT. Yet, the presence of thoroughly established toxicological and immunological profiles, the derivation from a natural resource, and the suitability and ease for manufacturing mass quantities would render LMWP to become the most preferable choice among current PTDs for important clinical applications, including molecular probes, protein, gene, and virtually all types of medical imaging and drug therapies. Aside from these direct clinical uses, the universal applicability of LMWP to transduce all types of compounds into cells, regardless their physical and chemical attributes (i.e., small or large, hydrophilic, or hydrophobic), could enable the discovery and/or development of new therapeutic or imaging agents, which are initially considered unusable as a result of their inability to enter the cells. In addition, the lack of sensitivity to serum (Fig. 2) and cell viability 
(Fig. 3), as demonstrated in this report, could allow LMWP to be used in the screening of potential therapeutic proteins, investigating protein-protein interaction in the cells, and elucidating the role of proteins in certain specific disease development. Taken together, naturally derived, nontoxic LMWP was suggested as a potential tool for carrying any biologic molecules to enhance the therapeutic or diagnostic efficacy.

\section{ACKNOWLEDGMENTS}

This work was supported in part by the National Institutes of Health Grant R01CA114612, and in part, by Korea Research Foundation Grant (KRF-2003-041-E00341), and by the International Collaboration Research Program by the Korean Science and Engineering Foundation (KOSEF, M6-0403-00-0019) through the Intellectual Biointerface Engineering Center at Seoul National University, Korea. The authors appreciate Chris Edward and Bruce Donohue (University of Michigan, Department of Cell and Development Biology, Medical Science Research Center) for technical assistance in conducting the confocal microscopy observation. The authors gratefully acknowledge Joseph Yang (Brown University, Providence, RI, USA) for his assistance in reviewing the manuscript preparation.

\section{REFERENCES}

1. Fawell, S., Seery. J., Daikh, Y., Moore, C., Chen, L. L., Pepinsky, B., and Barsoum, J. (1994) TAT-mediated delivery of heterogeneous proteins into cells. Proc. Natl. Acad. Sci. USA 91, 664-668

2. Snyder, E. L., and Dowdy, S. F. (2001) Protein/peptide transduction domains: potential to deliver large DNA molecules into cells. Curr. Opin. Mol. Ther. 3, 147-152

3. Mann, D. A., and Frankel, A. D. (1991) Endocytosis and targeting of exogenous HIV-1 TAT protein. EMBO J. 10, 1733-1739

4. Joliot, A. H., Pernelle, C., Deagostini-Bazin, H., and Prochiantz, A. (1991) Antennapediahomeobox peptide regulates neural morphogenesis. Proc. Natl. Acad. Sci. USA 88, $1864-1870$

5. Elliott, G., and O'Hare, P. (1997) Intracellular trafficking and protein delivery by a herpes virus structural protein. Cell 88, 223-233

6. Vives, E., Brodin, P., and Lebleu, B. (1997) A truncated HIV-1 Tat protein basic domain rapidly translocates through the plasma membrane and accumulates in the cell nucleus. $J$. Biol. Chem. 272, 16010-16017

7. Schwartz, S. R., Ho, A., Vocero-Akbani, A., and Dowdy, S. F. (1999) In vivo protein transduction: delivery of a biologically active protein into the mouse. Science $\mathbf{2 8 5}, 1569$ 1572

8. Josephson, L., Tung, C. H., Moore, A., and Weissleder, R. (1999) High efficiency intracellular magnetic labeling with novel superparamagnetic-TAT peptide conjugate. Bioconjug. Chem. 10, 186-191 
9. Lewin, M., Carlesso, N., Tung, C. H., Tang, X. W., Cory, D., Scadden, D. T., and Weissleder, R. (2000) Tat-peptide-derivatized magnetic nanoparticles allow in vivo tracking and recovery of progenitor cells. Nat. Biotechnol. 18, 410-414

10. Torchilin, V. P., Rammohan, R., Weissig, V., and Levchenko, T. (2001) TAT peptide on the surface of liposomes affords their efficient intracellular delivery even at low temperature and in the presence of metabolic inhibitors. Proc. Natl. Acad. Sci. USA 798, 8786-8791

11. Niesner, U., Halin, C., Lozzi, L., Gunthert, M., Neri, P., Wunderli-Allenspach, H., Zardi, L., and Neri, D. (2002) Quantification of the tumor targeting properties of antibody fragments conjugated to cell-permeating HIV-1 TAT peptides. Bioconjug. Chem. 13, 729-736

12. Futaki, S., Suzuki, T., Ohashi, W., Yagami, T., Tanaka, S., Ueda, K., and Sugiura, Y. (2001) Arginine-rich_peptides. J. Biol. Chem. 276, 5836-5840

13. Byun, Y., Singh, V. K., and Yang, V. C. (1999) Low molecular weight protamine: a potential nontoxic heparin antagonist. Thromb. Res. 94, 53-61

14. Chang, L. C., Lee, H. F., Yang, Z. Q., and Yang, V. C. (2001) Low molecular weight protamine (LMWP) as nontoxic heparin/low molecular weight heparin antidote (I): preparation and characterization. AAPS PharmSci. 3, E17

15. Chang, L. C., Liang, J. F., Lee, L. M., and Yang, V. C. (2001) Low molecular weight protamine (LMWP) as nontoxic heparin/low molecular weight heparin antidote (II): in vitro evaluation of efficacy and toxicity. AAPS PharmSci. 3, E18

16. Lee, L.M., Chang, L.C., Wrobleski, S., Wakefield, T.W., and Yang, V.C. (2001) Low molecular weight protamine as nontoxic heparin/low molecular weight heparin antidote (III): preliminary in vivo evaluation of efficacy and toxicity using a canine model. AAPS PharmSci. 3, E19

17. Tsui, B., Singh, V. J., Liang, J. F., and Yang, V. C. (2001) Reduced cross-reactivity towards anti-protamine antibodies of a low molecular weight protamine analogue. Thromb. Res. 101, 417-420

18. Liang, J. F., Zhen, L., Chang, L. C., and Yang, V. C. (2003) A less toxic heparin antagonistlow molecular weight protamine. Biochemistry (Mosc.) 68, 116-120

19. Park, Y. J., Liang, J. F., Ko, K. S., Kim, S. W., and Yang, V. C. (2003) Low molecular weight protamine as an efficient and nontoxic gene carrier: in vitro study. J. Gene Med. 5, 700-711

20. Carlsson, J., Drevin, H., and Axen, R. (1978) Protein thiolation and reversible proteinprotein conjugation. Biochem. J. 173, 723-737

21. Heeremans, J. L., Kraaijenga, J. J., Los, P., Kluft, C., and Crommelin, D. J. (1992) Development of a procedure for coupling the homing device glu-plasminogen to liposomes. Biochim. Biophys. Acta 1117, 258-264 
22. Mellman, I. (1996) Endocytosis and molecular sorting. Annu. Rev. Cell Dev. Biol. 12, 575625

23. Morris, M. C., Depollier, J., Mery, J., Heitz, F., and Divita, G. (2001) A peptide carrier for the delivery of biologically active proteins into mammalian cells. Nat. Biotechnol. 19, 11731176

24. Tung, C. H., Mueller, S., and Wessleder, R. (2002) Novel branching membrane translocational peptide as gene delivery vector. Bioorg. Med. Chem 10, 3609-3614

25. Zaro, J. L., and Shen, W-C. (2003) Quantitative comparison of membrane transduction and endocytosis of oligopeptides. Biochem. Biophys. Res. Commun. 307, 241-247

26. Rothbard, J. B., Kreider, E., VanDeusen, C. L., Wright, L., Wylie, B. L., and Wender, P. A. (2002) Arginine-rich molecular transporters for drug delivery: role of backbone spacing in cellular uptake. J. Med. Chem. 45, 3612-3618

27. Veenendaal, L. M., Jin, H., Ran, S., Cheung, L., Navone, N., Marks, J. W., Waltenberger, J., Thorpe, P., and Rosenblum, M. G. (2002) In vitro and in vivo studies of a VEGF $121 /$ gelonin chimeric fusion toxin targeting the neovasculature of solid tumors. Proc. Natl. Acad. Sci. USA 99, 7866-7871

28. Selbo, P. K., Sivam, G., Fodstad, O., Sandvig, K., and Berg, K. (2001) In vivo documentation of photochemical internalization, a novel approach to site-specific cancer therapy. Int. J. Cancer 92, 761-766

29. Rosenblum, M. G., Shawver, L. K., Marks, J. W., Brink, J., Cheung, L., and LangtonWebster, B. (1999) Recombinant immunotoxins directed against the c-erb-2/HER2/neu oncogen product: in vitro cytotoxicity, pharmacokinetics and in vivo efficacy studies in xenograft models. Clin. Cancer Res. 5, 865-874

30. Nagahara, H., Vocero-Akbani, A. M., Snyder, E. L., Ho, A., Latham, D. G., Lissy, N. A., Becker-Hapak, M., Ezhevsky, S. A., and Dowdy, S. F. (1998) Transduction of full-length TAT fusion proteins into mammalian cells: TAT-p27Kip1 induces cell migration. Nat. Med. 4, 1449-1452

31. McGrath, M. S., Rosenblum, M. G., Philips, M. R., and Scheinberg, D. A. (2003) Immunotoxin resistance in multidrug resistant cells. Cancer Res. 63, 72-79

32. Wender, P. A., Mitchell, D. J., Pattabiraman, K., Pelkey, E. T., Steinman, L., and Rothbard, J. B. (2000) The design, synthesis, and evaluation of molecules that enable or enhance cellular uptake: peptoid molecular transporters. Proc. Natl. Acad. Sci. USA 97, 13003-13008

33. Suzuki, T., Futaki, S., Niwa, M., Tanaka, S., Ueda, K., and Sugiura, Y. (2002) Possible existence of common internalization mechanisms among arginine-rich peptides. J. Biol. Chem. 277, 2437-2443

Received November 16, 2004; accepted May 19, 2005. 
Fig. 1
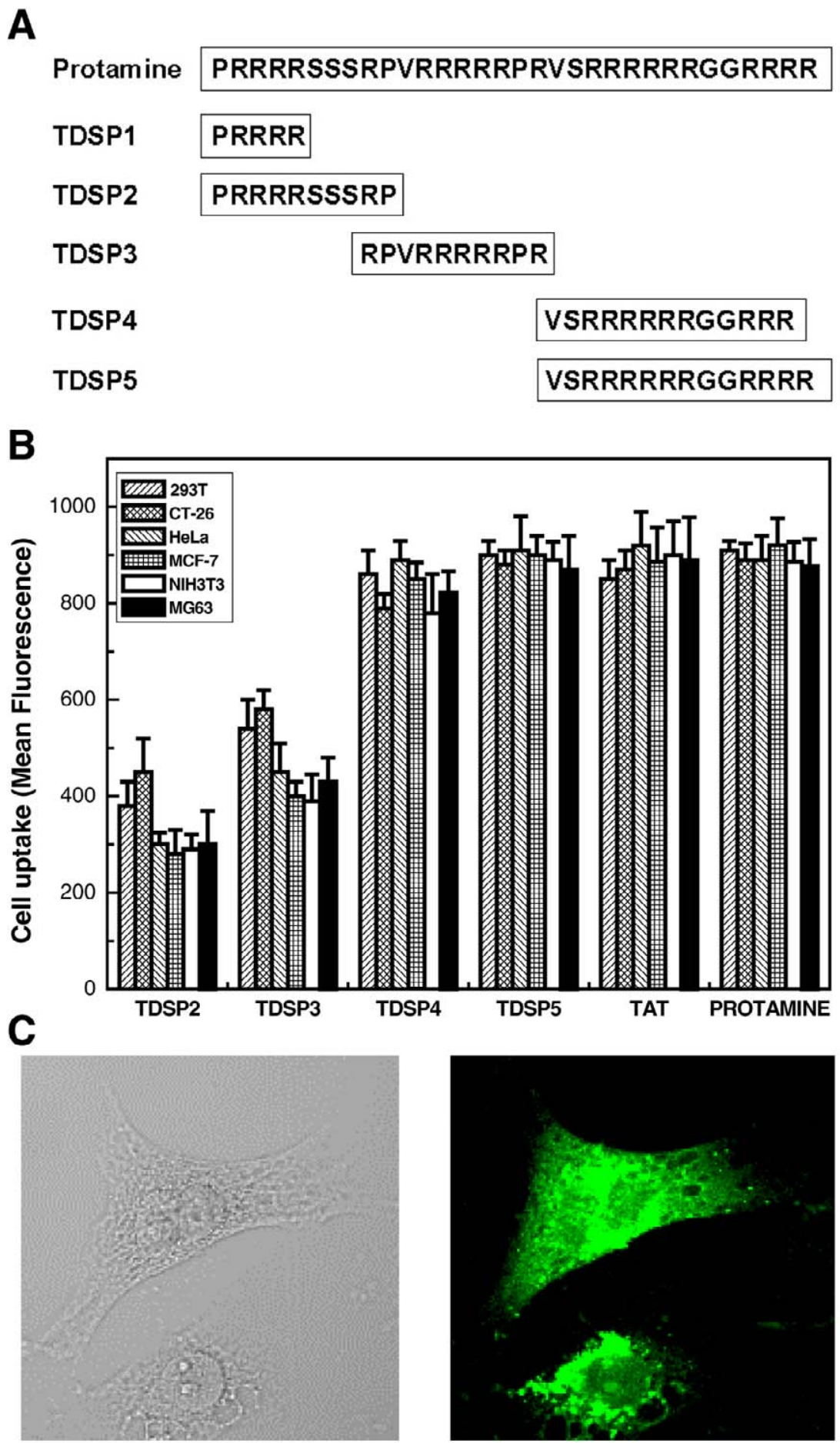

Figure 1. A) The amino acid sequence of LMWPs, which were prepared by digesting protamine with enzyme thermolysin. In accordance with the elution order from liquid chromatography, the LMWPs were denoted as TDSP1-5. B) Cell uptake of LMWPs. FITC-labeled LMWPs $(0.2 \mu \mathrm{M})$ were monitored in different cell lines including 293T, CT-26, HeLa, MCF-7, NIH3T3, and MG63. TAT peptide and protamine were served as controls to compare the transduction efficiency with LMWPs. FITC-labeled peptides were applied onto each group of cells $\left(1 \times 10^{6} \mathrm{cells} / \mathrm{well}\right)$ grown in the presence of $10 \%$ serum for $30 \mathrm{~min}$ at $37^{\circ} \mathrm{C}$. Cell uptake was determined by counting fluorescence with fluoresceinactivated cell sorter (FACS) analysis. $\boldsymbol{C}$ ) Cellular localization of TDSP5 peptide. FITC-labeled TDSP5 (0.2 $\mu$ M) was applied to HeLa cells grown to $75 \%$ confluency on a Lab-Tek ${ }^{\circledR}$ chamber coverglass in the presence of $10 \%$ serum for 30 min at $37^{\circ} \mathrm{C}$. Cellular localization was monitored by confocal microscopy. Left panel indicates the differential interference contrast (DIC) mode picture of TDSP5-treated HeLa cells, whereas the right panel is the identical fluorescent image. 
Fig. 2

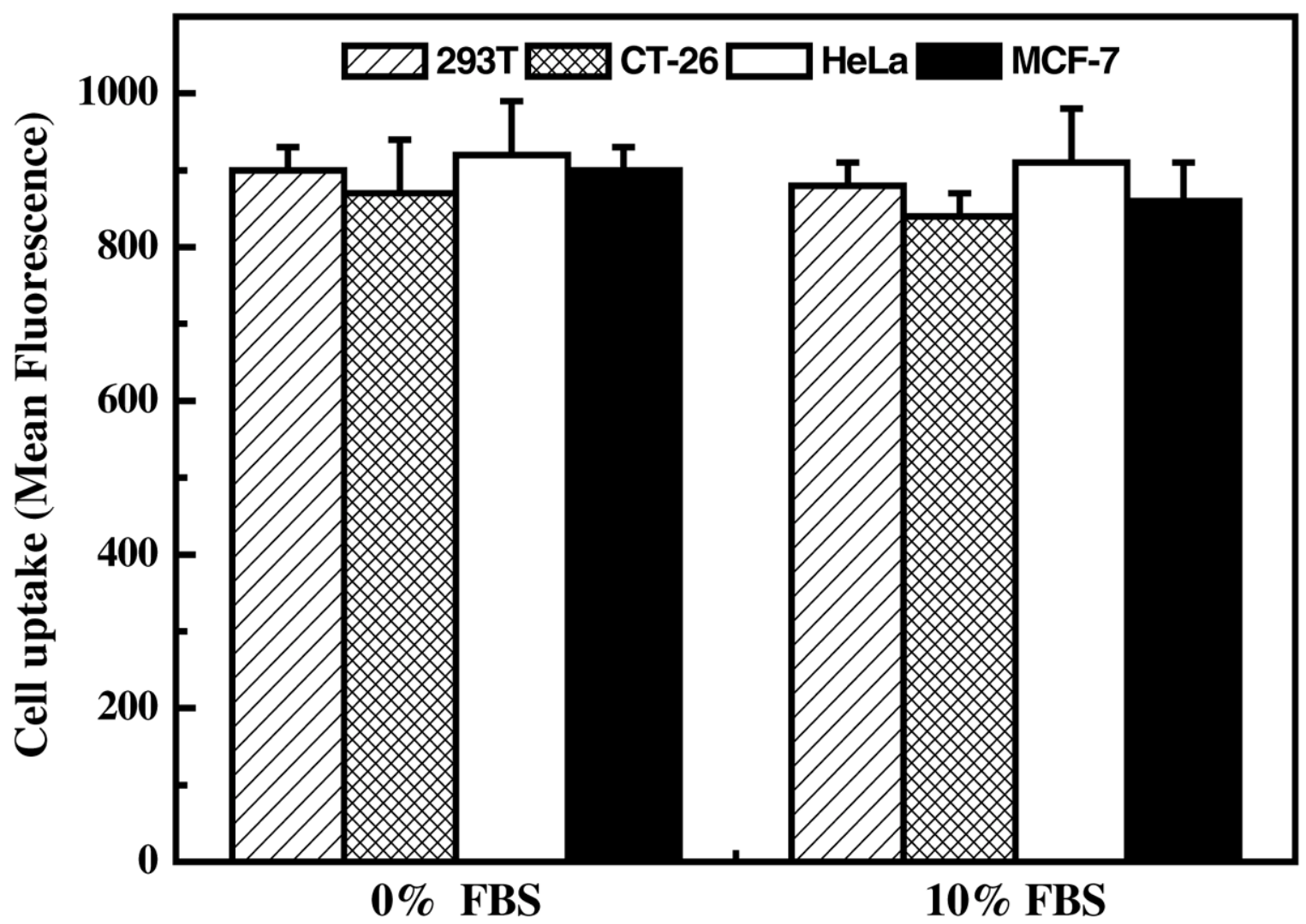

Figure 2. LMWP (TDSP5) internalization in different cell lines. FITC-labeled TDSP5 $(0.2 \mu \mathrm{M})$ was incubated with cells $\left(1 \times 10^{6}\right.$ cells/well $)$ in serum-free or $10 \%$ serum containing cell culture medium for $30 \mathrm{~min}$. Cells were then washed and detached, and their fluorescence was examined by FACS analysis (see Materials and Methods). 
Fig. 3

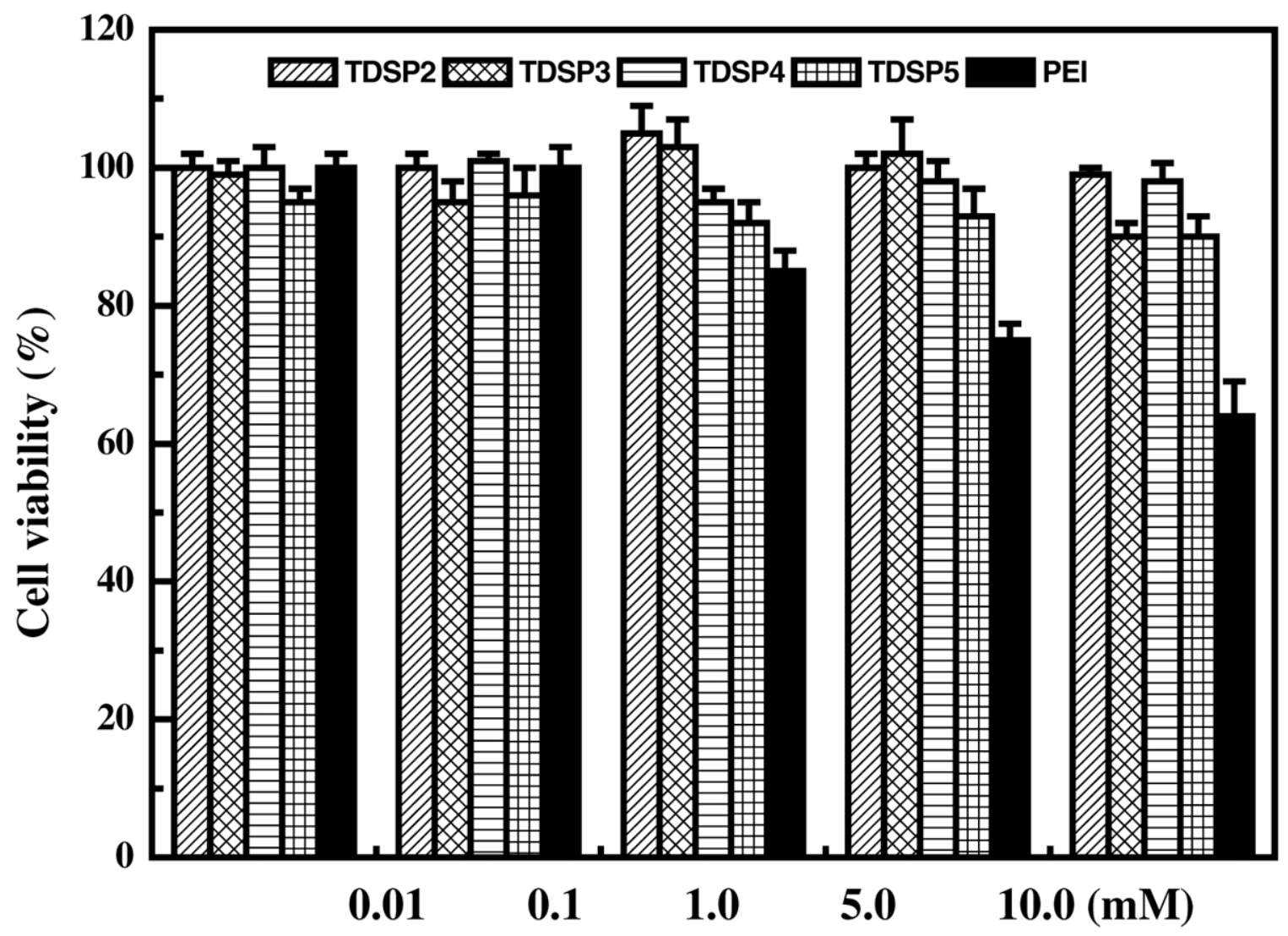

Figure 3. Cytotoxicity of LMWPs. The toxicity was monitored in $293 \mathrm{~T}$ cell lines by varying the concentration of each peptide including TDSP2, $-3,-4$, and -5 with TAT $_{47-57}$ as a control. Cell viability was quantified by MTT staining (See Materials and Methods) after 2 days of incubation. 
Fig. 4

A

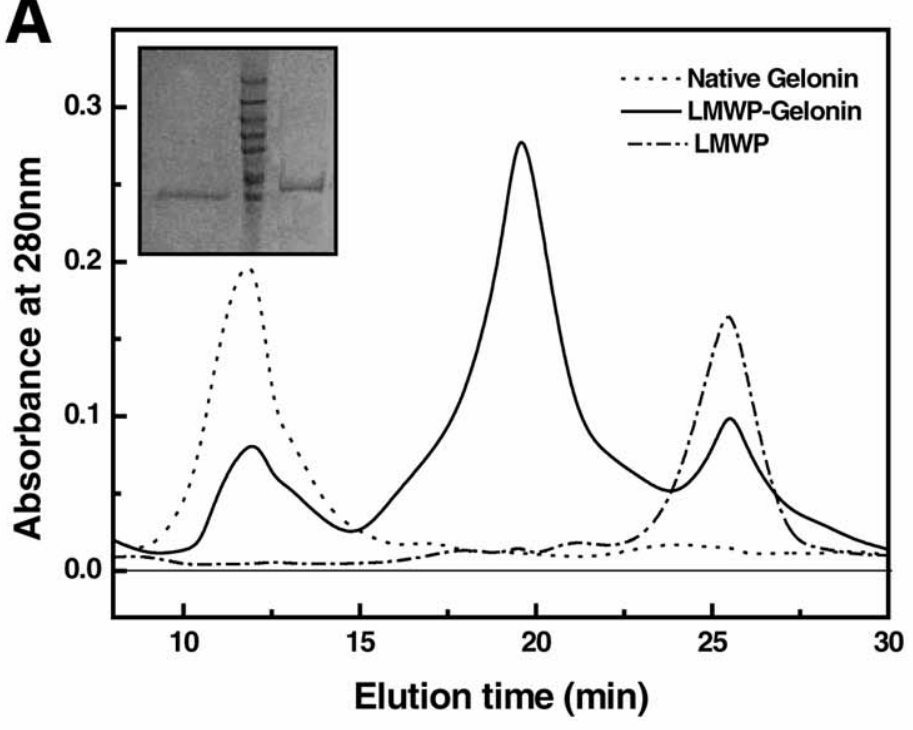

C Gelonin
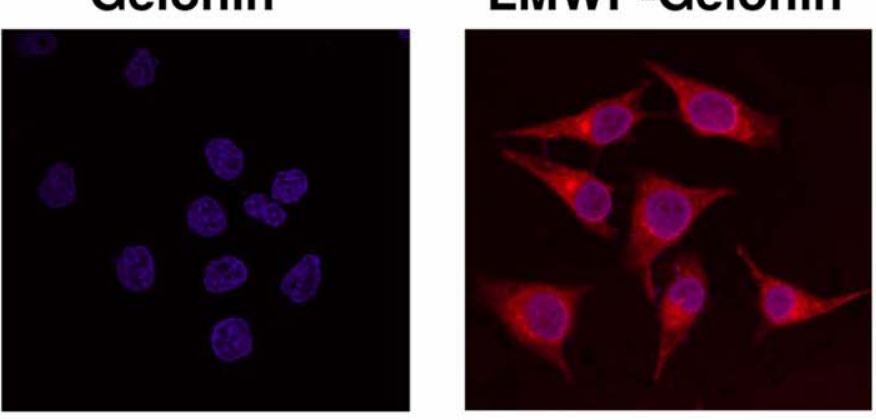

B

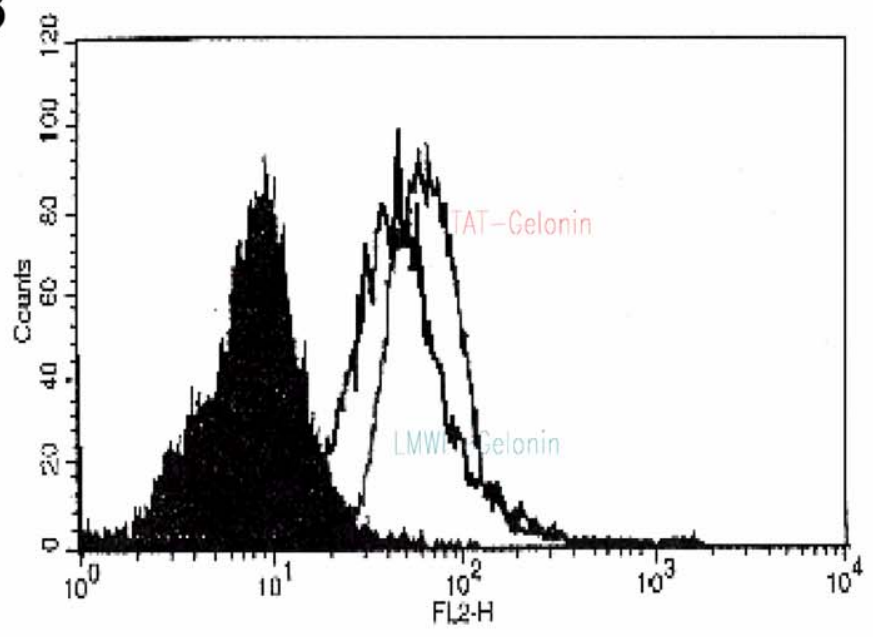

TAT-Gelonin

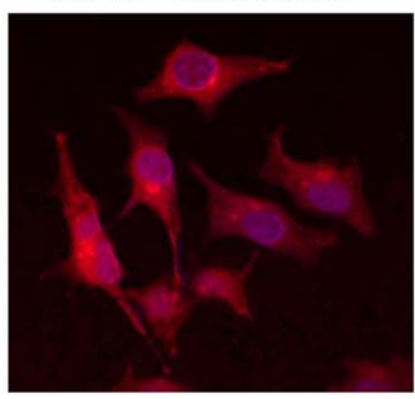



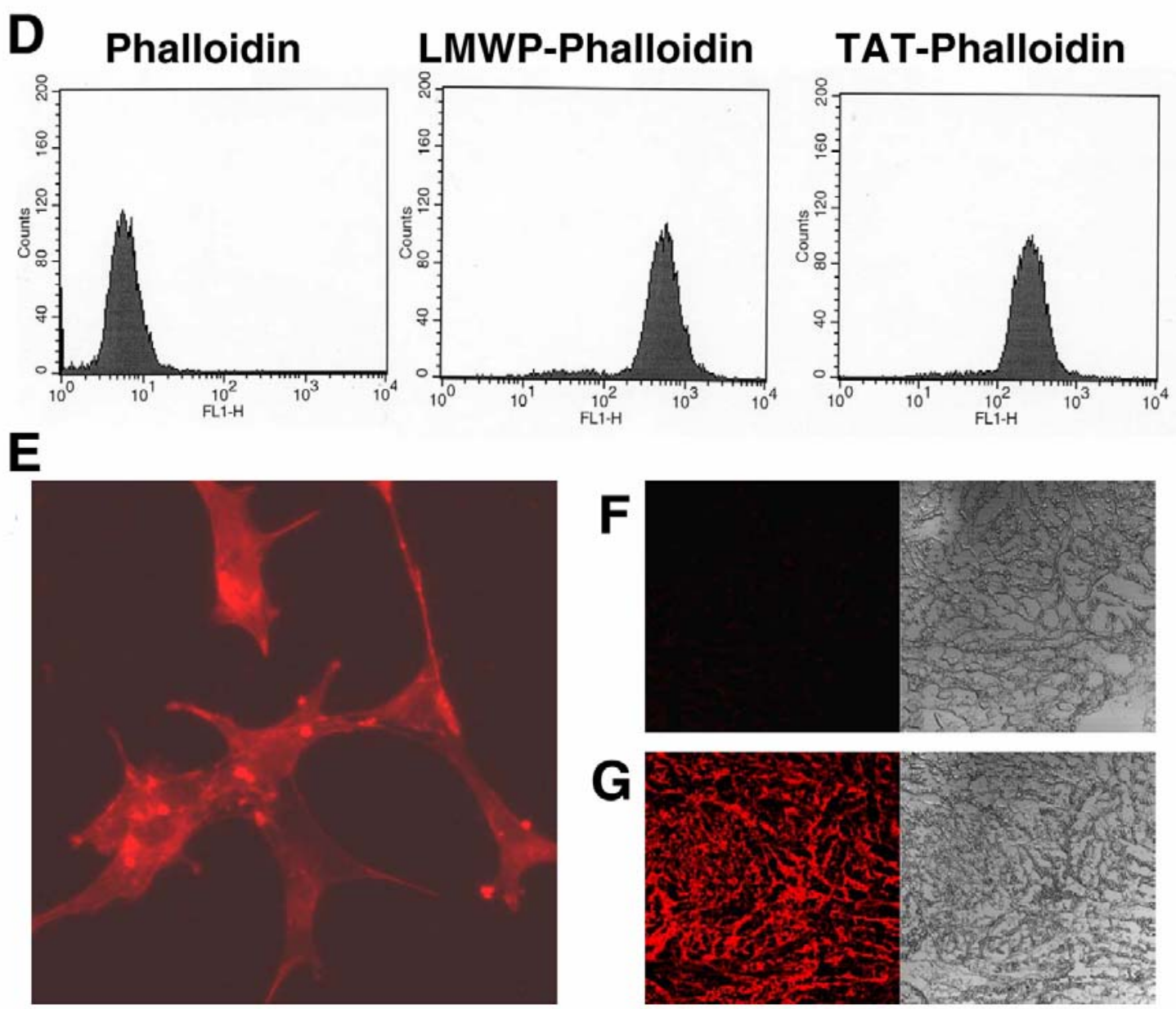

Figure 4. A) Formation of LMWP-gelonin conjugates. Prepared LMWP (TDSP5)-gelonin (see Materials and Methods) conjugate was purified from unconjugated gelonin and LMWP by eluting through a heparin-affinity chromatography column. Elution profiles were followed by absorbance at $280 \mathrm{~nm}$. Inset of the figure shows molecular weight of LMWPgelonin conjugates by SDS-PAGE analysis. LMWP-gelonin conjugate and native gelonin were examined by SDS-PAGE on a $12 \%$ gel, stained with Coomassie brilliant blue. The left lane of the molecular marker indicates the molecular weight of gelonin ( 29,000 Da), whereas the right indicates LMWP-gelonin ( 31,000 Da). The molecular weight marker ranges from 29,000 to 700,000 . B) FACS analysis of cell uptake by rhodamine-labeled LMWP-gelonin conjugate and rhodamine-labeled TAT-gelonin conjugate in a CT-26 cell line $\left(1 \times 10^{6}\right.$ cells/well $)$ in the presence of $10 \%$ serum. $\left.\boldsymbol{C}\right)$ Cellular localization of rhodamine-labeled LMWP-gelonin conjugates in CT-26 colon adenocarcinoma cells. Rhodaminelabeled gelonin, LMWP-gelonin conjugate, and TAT-gelonin conjugate were overlaid onto cultured CT-26 cells in the presence of $10 \%$ FBS. Cellular localization was monitored by confocal microscopy. D) FACS analysis of cell uptake by Alexa Fluoro488-labeled LMWP-phalloidin conjugate and TAT-phalloidin conjugate in MG63 osteoblast cells $\left(1 \times 10^{6}\right.$ cells/well) in the presence of $10 \%$ serum. Alexa Fluoro488-labeled phalloidin served as control. E) Cellular localization of rhodamine-labeled LMWP-phalloidin conjugates in MG63 osteoblast cells. Rhodamine-labeled LMWP-phalloidin conjugate was overlaid onto cultured MG63 cells in the presence of 10\% FBS. Cellular localization was monitored by confocal microscopy. F) Tumor penetration of rhodamine-labeled free gelonin into the colon cancer and $(\boldsymbol{G})$ rhodaminelabeled LMWP-gelonin conjugate. The mice were then analyzed for penetration extent with LMWP-gelonin conjugate through the tumors, which were isolated at $10 \mathrm{~h}$ after injection of rhodamine-labeled free gelonin or rhodamine-labeled LMWP-gelonin conjugate, and cryo-sectioned, and pictures were taken by confocal microscopy. The left panel indicates the fluorescent image of rhodamine-labeled protein transduced in the tumor tissue, whereas the right panel was an identical DIC mode picture. 
Fig. 5

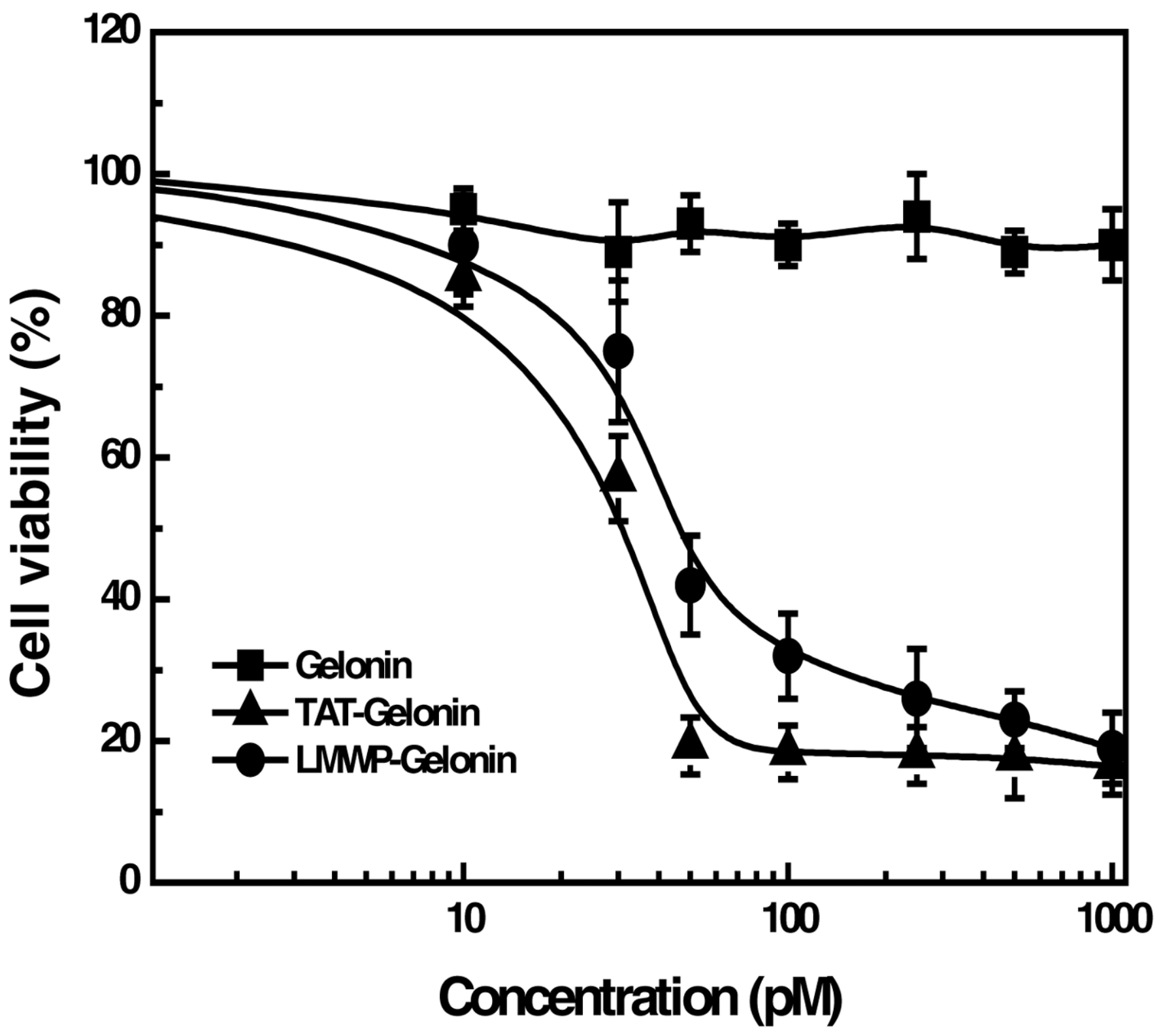

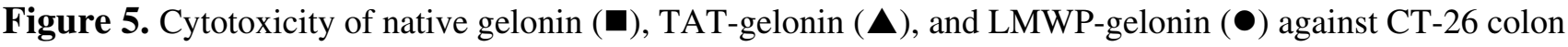
adenocarcinoma cells in log-phase culture. Various doses of each compound were added to the 96-well culture plates containing 5000 cells/well. The plates were incubated for $48 \mathrm{~h}$ at $37^{\circ} \mathrm{C}$ under an atmosphere of $5 \% \mathrm{CO}_{2}$ in humidified air in an incubator. The amount of remaining cells in the wells was assessed by MTT assay and then compared with those of untreated cells in the control wells. Values were represented as mean \pm SD. Each experiment was performed in triplicate. 
Fig. 6
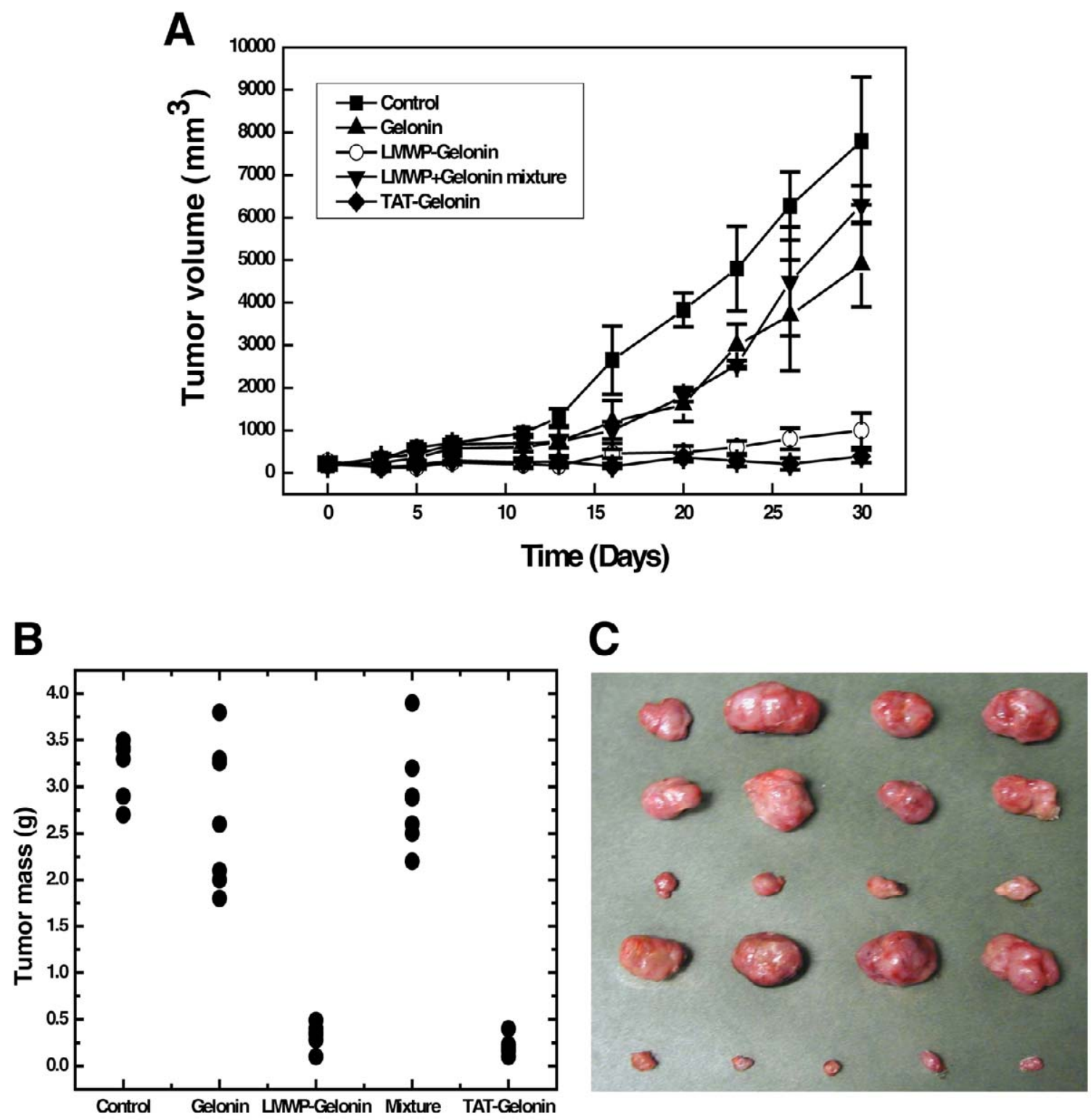

Figure 6. A) BALB/c mice received s.c. implants of the adenocarcinoma colon cancer cell line CT-26 in the right hind flank. Two weeks after implantation, the mice were divided randomly into groups of eight and treated with PBS, $100 \mu \mathrm{g}$ gelonin, $110 \mu \mathrm{g}$ LMWP-gelonin (equivalent to $100 \mu \mathrm{g}$ gelonin), $10 \mu \mathrm{g}$ LMWP and free gelonin mixture, and $110 \mu \mathrm{g}$ TATgelonin (equivalent to $100 \mu \mathrm{g}$ gelonin), via peritumoral injection. Tumor volume was determined by caliper measurements taken twice per week and averaged for each treatment group (see Materials and Methods). B) Peritumoral delivery of each sample. Peritumoral injection of LMWP-gelonin or TAT-gelonin conjugates around s.c. tumors in mice resulted in substantial decrease of tumor mass compared with tumors treated with samples including PBS, gelonin, and the mixture of gelonin and LMWP. Note the "Mixture" in the figure indicates the mixture of gelonin and LMWP. $C$ ) Peritumoral antitumor effects of PBS (Control), $100 \mu \mathrm{g}$ gelonin, $110 \mu \mathrm{g}$ LMWP-gelonin (equivalent to $100 \mu \mathrm{g}$ gelonin), $10 \mu \mathrm{g}$ LMWP and free gelonin mixture, and $110 \mu \mathrm{g}$ TAT-gelonin (equivalent to $100 \mu \mathrm{g}$ gelonin). Representative excised tumors from treated mice. The top to bottom represent tumors excised from mice treated with PBS solution (average tumor mass: $3.16 \pm 0.65 \mathrm{~g}) ; 100 \mu \mathrm{g}$ gelonin $(2.62 \pm 0.53 \mathrm{~g}) ; 110 \mu \mathrm{g}$ LMWP-gelonin $(0.33 \pm 0.12 \mathrm{~g}) ; 10 \mu \mathrm{g}$ LMWP and $100 \mu \mathrm{g}$ gelonin mixture $(2.74 \pm 0.68 \mathrm{~g})$; and $110 \mu \mathrm{g}$ TAT-gelonin $(0.21 \pm 0.04 \mathrm{~g})$. Samples were administered by peritumoral injections, which were started 2 weeks after tumor cell implantation when tumors reached $\sim 100 \mathrm{~mm}^{3}$, and mice were killed after 30 days after the initial treatment. The tumors were excised from mice and weighed, and photographs were taken. 